\title{
Relativity and View Effects
}

\author{
Bernard A. Fischli
}

Summary

This paper describes a new interpretation of relativity.

The concept of rest frames clarifies the initial assumptions used in General Relativity and underlines the necessity of a review of whatever impacts relativity. View effects are examined in addition to interactions. The Ehrenfest paradox is solved. View effects specific to each point of view are the solution. A curved space cannot be compatible with relativity. Both the line of sight and the path of the object are contracted. The calculations are based on path speeds at path points independently of speed orientation and direction. Relativistic clock time rates remain as previously detailed in relativity. Special Relativity is mainly reduced to the examination of Lorentz boosts. The concept of a seen speed is introduced. A seen speed in a Lorentz boost can reach infinity. Examples of view effects are discussed. Of importance is the path of stars seen from earth.

The calculation of the deflection of light by the sun explains in detail why the deflection angle must be almost double the value obtained with Newton's laws. As already noted by Einstein the relativistic contribution to the deflection can only be explained by an accelerated photon. This is a consequence of the view effects.

The compatibility of General Relativity with the new interpretation is discussed. The main argument for this compatibility is due to the use in General Relativity of a Pseudo-Riemannian geometry describing intrinsic views that are compatible with view effects. Several differences subsist.

Relativistic energy is examined. The empirical evidence only confirms formulas specific to electromagnetism and in particular the Lorentz factor used for the calculation of view effects. The paths of stars seen from the earth are not compatible with the interpretation of energy by Special Relativity. Relativistic paths are impacted by view effects that do not convey energy. Total energy is composed of rest mass energy plus a kinetic energy as defined in classical mechanics. The transmission of information is limited to the speed of light. An object has no speed limit due to relativity but can be limited due to other causes as is the case for photons. Electromagnetism describes a mixture of interactions and view effects but was interpreted as if it was specific only to interactions. The new interpretation impacts the analysis of empirical evidence. Dark energy should be calculated without relativistic contributions to energy.

Inertial behavior is due to energy exchanges originating from interactions and impacting the kinetic energy. Inertial masses are redundant, but this does not introduce gravitation to General Relativity.

\section{On inertial reference frames}

Galileo Galilei introduced the notion of inertial reference frames, to be called inertial frames, where the laws of motion could be valid in a Euclidian space. Newton proposed an absolute inertial frame in uniform motion relative to the stars. Einstein discarded the notion of an absolute inertial frame and developed Special Relativity that describes space transformations keeping the speed of light constant while maintaining the validity of the laws of motion in inertial frames. Inertial frames, as defined in Newton's first law, are reference frames where bodies either remain at rest or move at constant velocity unless acted upon by a force. 
This paper is restricted to relativity which is best explained starting from the non-relativistic description of interactions. This does not apply to electromagnetism as Maxwell's equations implicitly include relativistic effects making it more difficult to sort out the impact of relativity. Interactions valid in a Euclidian space such as classical mechanics and Newton's gravitation laws, to be called Newton's laws, will be further examined with an emphasis on gravitation.

Electromagnetism will be analyzed when reviewing energy.

In this paper an observer is a person or an equipment registering positions and movements in space as recorded by instruments such as an eye or a sensor of a camera or of a telescope or of whatever can record pictures of the impact of light waves or gravitational waves. For what follows "light waves" shall include "gravitational waves". An observer always observes out of his own rest reference frame, to be called a rest frame. A rest frame $\{x, y, z\}$ is in all circumstances an inertial frame relative to a chosen reference, in this case the observer. Therefore, the laws of motion of classical mechanics and Newton's laws are valid in the rest frame of the observer. What could be the use of inertial frames not matching the rest frame of the observer? They would represent inertial frames from which no observations would be made.

The observer sees an object. The words seeing and viewing are here synonymous to recording. An object is the subject of the information recorded by an observer, for instance on a photon, a black hole neighborhood, a car, a point on a wall or whatever else.

The observer and the object are represented by their rest frames and the laws of motion describe the relative motions and positions of these rest frames as seen by the observer. Both the observer and the object are located at the origins of their rest frames.

In Special Relativity (1) the rest frame of the observer is $\left\{x^{\alpha}\right\}=\{c t, x, y, z\}$ and the rest frame of the object is $\left\{\boldsymbol{X}^{\alpha}\right\}=\{c \tau, \boldsymbol{X}, \boldsymbol{Y}, \boldsymbol{Z}\}$

$\mathrm{t}$ is the times of the clock of the observer and $\tau$ is the time of the clock of the object, $\mathrm{c}$ is the speed of light.

In its rest frame the object has always the position $\{c \tau, 0,0,0\}$

Then $\frac{d^{2} \boldsymbol{X}^{\alpha}}{d p^{2}}=0$

where $\mathrm{p}$ is an affine parameter, for example the time $\tau$.

Equation [1] is valid in space and time and can be rewritten as:

$\frac{d^{2} \boldsymbol{X}^{\alpha}}{d p^{2}}=\frac{d}{d p}\left(\frac{\partial \boldsymbol{X}^{\alpha}}{\partial x^{\mu}} \frac{d x^{\mu}}{d p}\right)=0$

and results in the equation of General Relativity:

$\frac{d^{2} x^{\mu}}{d p^{2}}+\Gamma_{v \rho}^{\mu} \frac{d x^{\nu}}{d p} \frac{d x^{\rho}}{d p}=0$

with $\quad \Gamma_{v \rho}^{\mu}=\frac{\partial x^{\mu}}{\partial \boldsymbol{X}^{\alpha}} \frac{\partial^{2} \boldsymbol{X}^{\alpha}}{\partial x^{v} \partial x^{\rho}}$

$\Gamma_{v \rho}^{\mu}$ is called the Christoffel symbol. 
Equation [1] is valid for all points on the path of the object and equation [2] is specific to the same path.

Equation [2] results exclusively from the rest frame concept. Contrary to General Relativity (1) it depends neither on the equivalence principle nor on gravitation. At this stage of the theory there is no information on the sources impacting space and paths. The sources could be interactions and view effects. Interactions act using forces and by exchanges of energy. View effects act by deformations of seen space and paths without using forces or exchanges of energy. In what follows the interactions shall be as described in classical mechanics and Newton's laws.

A careful choice of the observer may simplify the calculations of interactions. When considering the deflection of light by the sun it is convenient to locate a virtual observer in the center of the sun and the observations shall be transmitted to the final observer usually located on earth.

\section{On the importance of the movements of the observer}

The importance of the movements of the observer relative to the observed object is explained by simple examples known under the name of aberrations.

Observer 1 and observer 2 are standing together. It starts raining and there is no wind. Both see the object, a raindrop, falling on a straight-line path pointing to the center of the earth due to gravitation. Observer 1 has an umbrella and stays in place. Observer 2 has no umbrella and decides to walk home at constant speed. He notices that his front side gets more wet than the back and understands that this is a consequence of his speed relative to the raindrops which now follow a straight-line path not pointing to the center of the earth. Observer 1 still sees the raindrops following straight-line paths pointing to the center of the earth.

Observer 2 gets more and more wet. He decides to run home. During the acceleration phase his speed relative to the raindrops progressively increases and so does the slope of his seen raindrop paths. Therefore observer 2 sees the raindrops following a curved path. Observer 1 still sees a straight-line path.

Observer 3 is the raindrop travelling next to the observed raindrop. They have a relative speed of zero. Observer 3 sees the other raindrop not moving at all.

The movements of an observer relative to an object can have a strong impact on the perceived path of the object and the laws of perceived motion of the object must incorporate that impact to predict the values recorded by an observer.

\section{On the Ehrenfest paradox}

The Ehrenfest paradox is about a disc rotating at constant angular velocity $\omega$ and whose circumference is subject to a relativistic contraction by a reciprocal Lorentz factor

$$
\frac{1}{\gamma}=\sqrt{1-\frac{\left(\omega r_{d}\right)^{2}}{c^{2}}}
$$

when observed from the center of the disc where $r_{d}$ is the uncontracted radius of the disc and $\mathrm{c}$ is the speed of light. In what follows the paradox will be considered a thought experiment with a totally rigid disc. The disc rotates in a room in the presence of furniture to which the reciprocal Lorentz contraction applies as well. 
A fixed observer does not rotate with the disc and a rotating observer rotates with the disc. Both are simultaneously located in the center of the disc. The fixed observer observes a contraction of the circumference of the disc but no contraction of the furniture whose speed relative to the fixed observer is zero. Simultaneously the rotating observer observes no contraction of the disc but a contraction of the furniture whose relative speed depends on the angular velocity of the rotational observer. No curved space can explain that. View effects can explain that.

The rotating observer sees a contracted table in the room. The fixed observer sees an uncontracted table. The rotation of an observer cannot contract a table, but view effects can contract the view of a table.

When the rotating observer sees different points on the table the local contraction specific to a point will depend on the speed of that point relative to the rotating observer and that speed depends on the relative distance to the point and on the angular velocity. Therefore, relativistic view contributions must be calculated for each point of view excepted for simple cases such as all the points on the circumference of the disc which are all subject to the same contraction.

A point on the radius $r_{d}$ of the disc is shared with the circumference of a circle of radius $r_{c}$ centered on the disc. As a curved space is excluded the circumference of that circle is equal to $2 \pi$ times the radius $r_{c}$ as observed from the center of the circle. Owing to the circular symmetry of that case a circle remains a circle after relativistic contractions and the proportionality of $2 \pi$ between the circumference and the radius remains valid.

The fixed observer sees each point on the radius $r_{d}$ moving at a different speed depending on its distance from the center of the disc. Using that speed, the contraction factor applicable at a point may be as calculated with equation [3]. A point close to the center would have a low path speed and contraction. A point at a greater distance to the center would have a higher path speed and a stronger contraction. An average of the contractions applying to the various points on the radius $r_{d}$ will always be smaller than the contraction applying to the circumference of the circle with radius $r_{d}$ thereby not respecting the proportionality factor of $2 \pi$.

A possible solution consists in introducing a factor specific to each circle and representing only the local contribution valid at the point common with the radius of the disc and applicable to the calculation of the overall contraction factor of a circle with radius $r_{d}$. The local contribution factor may be called the contribution factor of the circle with radius $r_{c}$.

The solution of the Ehrenfest paradox should respect the following conditions:

1) It should be compatible with view effects.

2) Relativistic view effects are due to the limitation in the speed of information transmission by light. This limitation is best accounted for by using somehow the Lorentz factor. A Lorentz factor as used in a Lorentz transformation guarantees a constant speed of light in an inertial frame.

3) The path speed of the object impacts the relativistic view effects. A higher speed results in a higher contraction.

4) The solution must respect the proportionality factor of $2 \pi$ between the circumference and the radius of any circular path contracted by a relativistic view effect.

The reciprocal Lorentz factor is proposed as a contribution factor. It could not be a contraction factor in this case as that would not respect condition 4). 
The overall contraction factor applicable to both the radius and the circumference of the disc, respecting thereby condition 4 ), is obtained by integrating the contribution factors over $r_{d}$ :

$\int_{0}^{r_{d}} \sqrt{1-\frac{\left(r_{c} \omega\right)^{2}}{c^{2}} d r_{c}}$

$r_{d}$ is the uncontracted radius of the disc.

$r_{c}$ is the uncontracted radius of a circle centered on the disc.

$\omega$ is the angular velocity of the disc.

$v_{c}$ is the uncontracted path speed of a point on the circle with radius $r_{c}$.

$v_{d}$ is the uncontracted path speed of a point on the circumference of the disc.

We have: $\omega=\frac{v_{d}}{r_{d}}$ and $v_{c}=r_{c} \omega=v_{d} \frac{r_{c}}{r_{d}}$ and $\beta=\frac{v_{d}}{c}$

$\int_{0}^{r_{d}} \sqrt{1-\frac{\left(\beta r_{c}\right)^{2}}{r_{d}^{2}}} d r_{c}$

The equation is solved by substituting $r_{c}$ with $x=\frac{r_{c}}{r_{d}} \beta$

and by substituting $\mathrm{x}$ with $\mathrm{u}$ and $\mathrm{x}=\sin (\mathrm{u})$

and using Euler's formula.

The contracted value of the disc radius is:

$r_{d}\left(\frac{1}{\beta}\left(\frac{\arcsin (\beta)}{2}+\frac{\sin (2 \arcsin (\beta))}{4}\right)\right)$

with $0 \leq \beta \leq 1$

\begin{tabular}{|c|c|}
\hline Path speed & Overall contraction factor \\
\hline Speed of light C & $\frac{\pi}{4}=0.7854$ \\
\hline$\frac{\pi}{12} \mathrm{C}=78^{\prime} 485^{\prime} 665$ meters per second & 0.988468 \\
\hline
\end{tabular}

The impact of the overall contraction factor is negligible up to speeds very close to the speed of light.

The prevailing interpretation of Special Relativity supposes no contraction of the radius of the disc. Why would the contraction be limited to the circumference of a circle? Point specific view effects solve the paradox. The disc itself never contracts.

What could be retained from Special Relativity? A reciprocal Lorentz factor as equation [3] is used for the determination of contribution factors and for the calculation of the contracted length of a straight-line path in a Lorentz boost. In what follows the contribution of Special Relativity will be mainly based on the examination of Lorentz boosts.

The straight-line going from the observer to the object shall be called a "line of sight". When using the rest frame concept with a Lorentz boost the observer is always located on the straight-line path of the object advancing at constant speed and that path also includes the line of sight. 
If the line of sight is also the path of the object and represents an accelerated path with the same speed distribution as for the radius of the Ehrenfest disc, then the overall contraction factor will be calculated with equation [4] as the local contribution factors will be given by reciprocal Lorentz factors specific to a path speed. These factors depend on the square of the speed and do not depend on speed direction. Therefore, the same overall contraction applies whether the speeds point towards the observer or away from him. In both cases the speeds have the same orientation as the path of the object. The path speeds for the Ehrenfest paradox disc are perpendicular to the radius that corresponds to the line of sight. For these three cases equation [4] applies to the calculation of the overall contraction factor independently of the orientations and directions of their path speeds. Due to the specificities of the contribution factors the same conclusions remain valid for any speed distribution simultaneously applied to the three cases. Therefore, the calculations are independent of the orientations and directions of the path speeds.

In general, the path has any shape and the observer is not located on the path.

- When looking at the object an observer will see the points on the line of sight moving because the object will move along its path thereby inducing a rotation of the line of sight as with the Ehrenfest paradox disc.

- The path speed of the object results from interactions and from movements of the observer relative to the object.

- As with the Ehrenfest paradox disc, that speed must be linearly reduced to give the speed valid as input for the calculation of the contribution factor at the concerned point on the line of sight.

- The contribution factors will impact the local contractions at the related points on the line of sight independently of speed orientation and direction. The overall contraction factor is then calculated using equation [4].

A high path speed induces a high overall contraction of the line of sight. This is how the deflection of light by the sun is impacted by a relativistic view effect.

We see the paths of the past as light needs time to transmit the information to the observer. We record the paths of the past including the impact of relativistic view effects that could be considered as aberrations of light in accelerated rest frames.

The important result is that the overall contraction factor of a line of sight depends on path speeds independently of speed orientation and direction.

\section{On time}

A local time difference is a measure of the size of a local change. In classical mechanics the local change is the infinitesimal segment of the path of an object corresponding to the infinitesimal time difference. The path of the object and the times seen and measured in the rest frame of the observer are described by the laws of interactions applying in inertial frames.

In Special Relativity a similar relationship applies to a Lorentz boost:

$d \tau^{2}=-\frac{1}{c^{2}} d s^{2}=d t^{2}-\frac{1}{c^{2}} d x^{2}$

$\tau$ being the time of the clock of the object and $t$ being the time of the clock of the observer.

$x$ is the space coordinate of the rest frame of the observer applying to the path of the object.

$d s^{2}$ is an interval between two events. An event in a four-dimensional reference frame $\{c t, x, y, z\}$ determines a unique position in space and time. 
A relative movement between clocks impacts the difference in clock rates:

$d \tau^{2}-d t^{2}=-\frac{1}{c^{2}} d x^{2}$

The following relationships apply to a Lorentz boost where we have $v=d x / d t$

$d \tau^{2}=d t^{2}-\frac{1}{c^{2}} d x^{2}=d t^{2}\left(1-\frac{v^{2}}{c^{2}}\right)=\frac{1}{\gamma^{2}} d t^{2}$

$v$ is the constant relative speed between the clock of the observer and the clock of the object as calculated in the rest frame of the observer before relativistic contractions.

$\gamma=\frac{1}{\sqrt{1-\frac{v^{2}}{c^{2}}}}$ is the Lorentz factor.

Equation [5] introduces a difference in clock rates due to a constant speed between two clocks with times $t$ and $\tau$. This effect is called velocity time dilation. When switching the roles of the observer and the object an observer's clock time will always be dilated respective to the clock time of the object as the calculations are done in the rest frame of the observer and therefore any impact on the clock of the observer will be assigned to a corresponding impact on the clock of the object.

Clock rates also depend on gravitational time dilation. A light wave travelling against a gravitational field is locally subjected to a redshift due to a Doppler effect as gravity and acceleration are equivalent in relativity. Furthermore, a relative speed of two clocks contributes to the relative redshift due to an additional Doppler shift factor in a gravitational field. The total redshift difference impacts the relative clock rate as calculated from the Doppler effects.

The clock nearest to the origin of the gravitational field will always have the slowest rate. This differentiation is possible because the redshift introduces an information on the orientation and direction of the electric field. Such information is missing with velocity time dilation that can therefore only deliver a clock rate difference that is independent of speed orientation and direction.

When measuring time differences between two clocks the clock rate differences will be due to the velocity and gravitational time dilations encountered during the respective trips of the clocks. A corresponding difference in clock times depends on the time spent with each value of a difference in clock rate as done in 1971 by Haefele and Keating (2) who recorded a time difference on two flights around the world and their measures matched predictions with an accuracy of about $10 \%$. The satellites of the Global Positioning System are the continuous empirical evidence that different clock rates must be accounted for as otherwise the system could not perform.

The expression used for the contribution factor of space contractions can be derived from velocity time dilation. We have from [5]:

$d \tau=\frac{1}{\gamma} d t$

The speed $v$ is calculated in the rest frame of the observer and is used to obtain both the uncontracted and the contracted infinitesimal segment lengths $d L$ and $d L_{c}$ in that rest frame:

$d \tau \cdot v=d L_{c} \quad$ and $\quad d t \cdot v=d L \quad$ therefore: $\quad d L_{c}=\frac{1}{\gamma} d L$

Contributions to clock rate differences and length contractions use the same contribution factor values when calculated for velocity time dilation. 
A time difference results from the impacts of the clock paths on each clock. The overall contraction factor of a line of sight is calculated from path speeds specific to each point on the line of sight and does not apply to time differences.

\section{On Lorentz boosts}

Relativity acts on space as a view effect. The initial conditions specific to each point on the path of the object and from which such effects are calculated originate from the laws of interactions combined with the contributions from the relative movements between the observer and the object.

As in classical mechanics an observer sees everything out of his rest frame which is a Euclidian space. The only impact of relativity on space is the relativistic contraction of the seen path that is embedded in the rest frame of the observer.

For a Lorentz boost the transformation rules between the rest frame of the observer and of the object are:

$x^{\prime}=\gamma(x-v t)$ and $d \tau=\frac{1}{\gamma} d t$

with $\gamma=\frac{1}{\sqrt{1-v^{2} / c^{2}}}$

$x$ is the coordinate value of an object as calculated in the rest frame of the observer before contractions due to view effects.

$x^{\prime}$ is the coordinate value of an object as seen by the observer in his rest frame after contractions due to view effects.

$v$ is the constant speed of an object as calculated in the rest frame of the observer before

contractions due to view effects with $v=d x / d t$ for a Lorentz boost.

$t, \tau$ and $c$ as previously defined

The information received by the observer is limited to the observation of the contracted path of the object. He does not know what the object's clock indicates and must use his own clock to calculate a "seen speed" $v_{s}$ of the object. The "seen speed" is the speed along the seen path as recorded by the observer.

In the case of a Lorentz boost the observer records the path of the object as a point and cannot "see" a related speed. Whenever the observer is not located on the path of the object, he registers angular movements and sees a succession of path points whose relative distances to himself are contracted by relativistic view effects as described in the section "On the Ehrenfest paradox". The values of the contraction factors are not recorded by the observer and must be evaluated by other methods.

The formula, valid for a path on the $x$-axis

$$
\frac{d x^{\prime}}{d t}=\gamma\left(\frac{d x}{d t}-v\right)=0
$$

gives the object's "seen speed" in the object's rest frame. The seen speed $v_{s}$ valid in the observer's rest frame is obtained by discarding the last term:

$v_{s}=\gamma \frac{d x}{d t}=\gamma v$ 
In Special Relativity the three space components $v_{f}^{\alpha}$ of the four velocity are defined as the division of the infinitesimal not contracted segments $d x^{\alpha}$, not seen by the observer, by the infinitesimal clock time $d \tau$, not received by the observer either. For a Lorentz boost the following formulas apply:

$v_{f}=\frac{d x}{d \tau}=\frac{d x}{d t / \gamma}=\gamma \frac{d x}{d t}=\gamma v=v_{s}$

The space component of the four velocity is identical to the seen speed.

What could be interpreted as the real speed is the speed $v$ as previously specified with, for a Lorentz boost:

$v=v_{s} \sqrt{1-\frac{v^{2}}{c^{2}}}=\sqrt{\frac{1}{\frac{1}{v_{s}^{2}}+\frac{1}{c^{2}}}}$

and $\quad v_{s}=\sqrt{\frac{1}{\frac{1}{v^{2}}-\frac{1}{c^{2}}}}$

with the following values:

\begin{tabular}{|c|c|}
\hline $\boldsymbol{v}$ & $\boldsymbol{v}_{\boldsymbol{s}}$ \\
\hline \hline $0.0995 \mathrm{C}$ & $0.1 \mathrm{C}$ \\
\hline $0.4472 \mathrm{C}$ & $0.5 \mathrm{C}$ \\
\hline $0.7071 \mathrm{C}$ & $\mathrm{C}$ \\
\hline $0.8944 \mathrm{C}$ & $2 \mathrm{C}$ \\
\hline $0.99995 \mathrm{C}$ & $10 \mathrm{C}$ \\
\hline $\mathrm{C}$ & $\infty$ \\
\hline
\end{tabular}

Values calculated for a Lorentz boost concern non-accelerated straight-line paths, with an observer located on the path and receiving the information on the positions of the object by light waves, limiting therefore the scope of equations [6] and [7].

That a seen speed of infinity may correspond to a path speed equal to the speed of light requires further examination detailed in the section "On energy and speed".

Relativistic view effects are specific to observations. When swapping the roles of the observer and the object a length contraction is always seen by the one chosen as observer.

\section{$\underline{6 . E x a m p l e s}$ of relativistic view effects}

A sphere will always be a sphere when subjected to view effects that will decrease the seen size but keep the shape. This result has already been described by the Terrell-Penrose effect (3).

Particle accelerators such as the CERN and Fermilab are examples of Lorentz boosts. The observers are the particle detectors placed on the path of the particles where particles rotating clockwise will collide with particles rotating anticlockwise when the requested speed will be reached. The particle path can be locally approximated by a straight-line. 
When an object advances at constant speed on a straight-line and the observer is not located on the path of the object, the relativistic contraction results in a parallel shift towards the observer. As per equation [4] the constant path speed applies the same overall contraction factor to all lines of sight and produces the shift.

Relativistic view effects depend on the speed of an object. That speed results from interactions and from the movements of the observer relative to the object. These movements contribute to the view effect by impacting the path speed used to calculate the contribution factors. An example is the rotation of the observer or of the disc as described for the Ehrenfest paradox. Observations made on earth include view effects due to the earth's rotation. The nearest star is Proxima Centauri at 4.244 light years and the contribution of the earth's rotation to the path speed is of 9740 times the speed of light.

Stars are observed on earth to move on a circular path due to the rotation. This cannot be explained by Special Relativity that assumes a contraction applying only to the object's path as well as a path contracted to a point in the center of the rest frame of the observer when the path speed reaches the speed of light. However, such a circular path is compatible with a contraction of the line of sight

by a factor of $\frac{\pi}{4}$ applying to the circular path as well.

A contribution of superluminal speeds (speeds above the speed of light) to the overall contraction of a line of sight cannot be recorded by an observer who sees the line of sight as a point. Angular movements remain unchanged. Furthermore, the telescopes for professional use are mounted since decades in a configuration with computerized tracking to compensate for rotations of any origin previously corrected by an equatorial mount.

\section{On the deflection of light by the sun}

Bending of light by the sun has been an iconic case establishing Einstein as the foremost scientist in the new relativistic world of the early $20^{\text {th }}$ century. The first calculations of the deflection angle date back to the $18^{\text {th }}$ century and were based on Newton's laws with gravitational forces bending the path of the light particle called photon. Travelling on a straight-line path, the photon is attracted to the sun but its high speed provides an escape after an exceedingly small deflection. A simple calculation approach is detailed in mathpages (4).

Expressed in a reference frame with the sun located in the center and the photon moving on an almost straight-line parallel to the $x$-axis with $y=r_{0}$ at $x=0, r_{0}$ being the radius of the sun, the path deviation of the photon is, when supposing a uniform rate of acceleration $a$ in the direction of the $y$ axis:

$$
y(t)=y_{0}+v_{0} t+\frac{1}{2} a t^{2}
$$

$t$ is the time

$y_{0}$ is the initial position

$v_{0}$ is the initial speed in the direction of the $y$ axis

For an almost constant speed of the photon the time can be approximated by

$$
t \approx \frac{x}{c}
$$

c is the speed of light in vacuum with a value of 299792458 meters per second 
We have:

$\frac{d y}{d x}=\frac{v_{0}}{c}+\frac{a}{c^{2}} x=\tan (\theta)$

$\theta$ is the angular coordinate with, for very small angles: $\tan (\theta) \cong \theta$

The gravitational acceleration calculated with Newton's law is:

$a=\frac{M G}{r^{2}} \frac{r_{0}}{r}$

where $M$ is the mass of the sun, $r$ is the distance from the center of the sun to the photon and $G$ is

the gravitational constant. The term $\frac{r_{0}}{r}$ is added to obtain the acceleration transverse to the path.

Then:

$\frac{d \theta}{d x}=\frac{d}{d x}\left(\frac{d y}{d x}\right)=\frac{a}{c^{2}}=\frac{M G r_{0}}{c^{2} r^{3}}$

With $r^{2}=r_{0}^{2}+x^{2}$ the deflection angle of the photon becomes:

$\int_{-\infty}^{+\infty} \frac{M G r_{0}}{c^{2}\left(r_{0}^{2}+x^{2}\right)^{1.5}} d x=\frac{2 M G}{c^{2} r_{0}}=0.8754$ seconds

mathpages goes on to a more rigorous approach based on a hyperbolic path with varying photon speeds. Compared to the previous simple calculation this adds corrections of about $2 * 10^{-6}$ seconds, a negligible amount.

As seen by the virtual observer located in the center of the sun the relativistic contribution to the deflection of light by the sun must match the non-relativistic path calculated with Newton's gravitation law and in particular the speed valid at each path point.

Given its sufficient accuracy, the same straight-line approach described above can be used to determine the relativistic contribution. A straight-line with path point speeds equal to those calculated for gravitation and seen by the same observer will be bent by a view effect to the same deflection angle and the same acceleration required to reach the same path speeds as with gravitation. The total seen deflection angle will therefore be double the angle calculated from Newton's laws up to factors of about order $10^{-5}$ due to the approximations used in the calculations. This result is compatible with the empirical evidence described in (5).

The acceleration of the photon induces changes in the contribution factors. As a contraction of the length of a straight-line path cannot bend a path by itself the bending is due to the contraction of the lines of sight. As the speed of light never reaches infinity any accelerated object advancing on a straight line is observed as advancing on a bent line due to a view effect.

Starting from the path due to an interaction and to the relative movement of the observer, the deflection angle can also be obtained by selecting a sufficient number of path points, calculating the contraction of each line of sight using equation [4] with the path speed valid at the considered point and interpolating between the contracted points. This method applies to any path with speeds of no more than the speed of light.

The additional deflection due to a view effect is a consequence of the fact that relativistic effects come on top of the initial conditions resulting from gravitation and that it can be calculated, up to 
negligible terms for this special case, as an additional bending of an accelerated straight-line path. This has the added advantage to explain the almost doubling of the deflection angle.

A more detailed description results from a decomposition of the hyperbolic path of the photon.

The impact parameter $b$ determines the shortest distance from the center of the sun, where the virtual observer is located, to each of the asymptotes of the hyperbola, with:

$b=-a \sqrt{\left(e^{2}-1\right)}$

$a$ is the semi-major axis that can be approximated up to a factor of $10^{-4}$ by $a=-\frac{\mu}{c^{2}}$

where $\mathrm{c}$ is the speed of light and $\mu$ is the standard gravitational parameter with, for the sun:

$\mu=1.3271244 * 10^{20} \frac{\mathrm{m}^{3}}{\mathrm{sec}^{2}}$

$e$ is the eccentricity with, as calculated in (6):

$e \cong \frac{c^{2} r_{0}}{G M}=4.711 * 10^{5}$ and $\mathrm{c}, \mathrm{G}, \mathrm{M}$ and $r_{0}$ as previously defined

The impact parameter has a calculated value of $6.951 * 10^{8}$ meters for both asymptotes, to be compared with a sun radius of $6.957 * 10^{8}$ meters.

The distance of closest approach is the periapsis distance $r_{p}$ with:

$r_{p}=a(1-e)=6.95638 * 10^{8}$ meters

As the radius of the sun, the impact parameter and the periapsis distance have almost the same value, the hyperbolic path of the photon can be described by the incoming asymptote up to its nearest point to the sun, to be called the impact point, then by a circular path ending at the impact point of the outgoing asymptote and finally by the outgoing asymptote. This is only possible as the hyperbolic path in an almost straight-line.

In gravitation the photon is accelerated in the straight-line path of the incoming asymptote and decelerated in the straight-line path of the outgoing asymptote. These accelerated and then decelerated straight-lines produce the relativistic contribution to the total deflection angle as described above.

In the circular path between the two impact points the force of gravitation is perpendicular to the path of the photon, keeping thereby the path speed constant. The circular path will be impacted by a constant contraction factor keeping after contraction the angle of deflection between the impact points as calculated from gravitation. The relativistic view effect for a circular path will only confirm the deflection value due to gravitation without adding any contribution.

Let us introduce the curved space paradox. The seen deflection of light by the sun is double the value calculated with Newton's laws. The usual interpretation is that the doubling results from a contribution of the curved space. According to Einstein, space is bent by the local energy content represented to a large extent by the rest mass of bodies. A photon has no rest mass. How could it bend space to such an extent as to double the deflection angle? The earth follows an elliptical path around the sun and has a considerable rest mass. Why does that mass have no impact on the path? 
The paradox is solved as the curved space does not exist. The relativistic impact is due to view effects resulting from the high speed of the photon. The orbital speed of the earth around the sun is too small to impact the contraction values. Furthermore, the earth follows around the sun an elliptical path with speeds varying by about $3.4 \%$ and a low eccentricity of 0.0167 corresponding to a path very close to a circle. For such a path with an almost constant speed a relativistic view effect will make only negligible contributions to the deflection angle of the earth as seen from the center of the sun whatever the path speed may be.

The speed of light in vacuum has always the same value when measured in inertial frames with constant relative speed. This does not apply to the rest frame of the photon which is accelerated due to gravitation as seen in the rest frame of the virtual observer.

Einstein wrote in his 1920 book on relativity (7):

Quote

...according to the general theory of relativity, the law of the constancy of the velocity of light in vacuo, which constitutes one of the two fundamental assumptions in the special theory of relativity and to which we have already frequently referred, cannot claim any unlimited validity. A curvature of rays of light can only take place when the velocity of propagation of light varies with position. Unquote

This is the case since a non-accelerated straight-line path is parallel shifted by a relativistic view effect without any bending of the path.

Variations in the speed of light are compatible with relativistic view effects as these can be calculated for any photon speed.

The orbital speed of the earth around the sun is if of about $29^{\prime} 780 \mathrm{~m} / \mathrm{s}$. It is too small to impact the deflection angle of the photon seen from earth. The final observer measures the difference between the angular position of the photon received directly from the source and the one after bending by the sun and view effects. The result is independent of the rotation of the earth and the deflection angle will be as seen by the virtual observer.

A photon has no rest mass. According to Newton's second law:

$f=m a$

with $f$ being the force, $m$ the mass and $a$ the acceleration, a photon may be accelerated to infinity at the slightest impact of a force. However, this equation can only be fully interpreted if the considered force is detailed in the equation.

The acceleration due to gravitation, calculated with Newton's laws, valid if one mass is much larger than the other and when observed from the larger mass $M_{0}$, is:

$a=\frac{G M_{0}}{r^{2}} \overrightarrow{r_{u}}$

$a$ is the acceleration of the small mass

$G$ is the gravitational constant

$r$ is the distance between the centers of the two masses

$\overrightarrow{r_{u}}$ is the unit vector in the direction of the larger mass

This is the acceleration used at the beginning of this section. It does not depend on the mass of a photon deflected by the sun. It depends only on the mass of the sun and on the distance to the center of the sun. 
A photon will never reach an infinite speed when deflected. On the path from the periapsis point to the final observer the photon is decelerated as it is attracted to the sun and will reach again the speed $\mathrm{c}$ valid in an inertial frame when the attraction to the sun becomes negligible.

\section{On General Relativity}

General relativity has the best matching of empirical evidence with values calculated for gravitation and is considered as a monument in physics. The possible drawback is its opacity which blurs the interpretation of the theory behind thick mathematical smoke. The curved space that justified the theory being invalidated; the question is raised whether the new interpretation could put the theory on more solid ground.

Einstein's famous equation of General Relativity is:

$E^{\mu \nu}=\frac{8 \pi G}{c^{4}} T^{\mu \nu}$

$E^{\mu v}$ is the Einstein tensor and $T^{\mu v}$ is the stress-energy tensor.

The Einstein tensor expresses the curvature of a pseudo-Riemannian manifold.

In General Relativity the approximations made in the calculations for weak and slowly variable fields systematically use a Newtonian potential (1) for gravitation. Why should this be necessary if that potential would only apply to find the value of the constant $\frac{8 \pi G}{c^{4}}$ at the non-relativistic limit and to incorporate it in Einstein's equation?

The same comment applies to the Schwarzschild metric of bodies whose mass distribution respects a spherical symmetry. The Schwarzschild metric is extensively used to describe the characteristics of black holes. Michell and Laplace had already proposed the existence of black holes in the late $18^{\text {th }}$ century. The description of gravitational waves also makes extensive use of Newtonian notions. All this is interpreted here as Einstein's choice of Newton's laws for gravitation with an added theoretical layer to account for relativistic effects.

The Einstein tensor was selected as it depends on derivatives of first and second order as is in general the case for the equations used in physics (9).

The main argument for the equivalence of the two approaches results from the use of a pseudoRiemannian geometry in General Relativity. That geometry is intrinsic. It describes what an observer located on any surface will see and measure in contrast to an extrinsic point of view where the observer is in a space of higher dimension. In other words, a pseudo-Riemannian geometry accounts for view effects of all sorts and is compatible with relativistic view effects. There is no particular reason why the validity of that geometry should be restricted to a curved space. It may apply as well to the description of curved paths.

The deflection of light by the sun calculated with General Relativity gives the correct result and that implies that the corresponding path and line of sight contractions must be compatible with the new interpretation.

Einstein's equivalence principle states that it is always possible to choose an inertial frame where the laws of nature are as given in Special Relativity possibly except for gravitation. The systematic use of Newton's gravitation laws implies that they apply as well. 
The new interpretation retains that the laws of nature apply to the Euclidian space of the rest frame of the observer instead of the Minkowski space of Special Relativity. A closer examination is required to understand how General Relativity works to still give the correct result for the deflection of light by the sun.

The four velocity $u^{\mu}$ of Special Relativity is defined as

$u^{\mu}=\frac{d x^{\mu}}{d \tau}$

with $\tau$ as previously defined.

The expression for $d \tau$ is somewhat different in General Relativity but reduces to the expression of Special Relativity in flat spacetime in which it has a value of zero when the path speed reaches the speed of light as per equation [5]. Therefore, the space coordinates of the four velocity, that can be interpreted as the seen speed, become infinite.

How can a path speed be the speed of light with an infinite seen speed? Is General Relativity impacted at all? Is so, what would be the consequences?

\section{On Energy and Speed}

The relativistic energy-momentum equation of an object is:

$E_{t}^{2}=m_{0}^{2} c^{4}+p^{2} c^{2}$

$E_{t}$ is the total energy or relativistic energy

$m_{0}$ is the rest mass of the object

$c$ is the speed of light

$p$ is the magnitude of the three-dimensional relativistic momentum of the object with:

$$
p^{i}=\frac{m_{0} v^{i}}{\sqrt{\left(1-\frac{v^{2}}{c^{2}}\right)}}
$$

$v^{i}$ is the space component $i$ of the speed $v$ as previously defined

The term $m_{0} c^{2}$ represents the energy content of the mass of the object at rest. The term $p^{2} c^{2}$ represents the contribution of relativistic kinetic energy. The relativistic kinetic energy is defined relative to an observer. The rest mass energy does not depend on an observer and is therefore not impacted by relativity.

The Lorentz transformation ascertains a constant speed of light in inertial frames. This particularity is the source of the relativistic view effects and is a consequence of Maxwell's equations.

Electromagnetism is a special case as Maxwell's equations are compatible with relativistic effects in addition to describing interactions.

Many experiments were carried out to confirm the relativistic relations using electromagnetic forces and measuring in particular the Lorentz factor $\gamma$ :

$$
\gamma=\frac{1}{\sqrt{\left(1-\frac{v^{2}}{c^{2}}\right)}}
$$


which applies to the calculation of the relativistic contraction of the radius of the Ehrenfest disc.

One of the most accurate if not the most accurate measurement was carried out by Meyer et al (8). The expression

$$
Y=\frac{m / m_{0}}{\sqrt{\left(1-\frac{p^{2}}{m_{0}^{2} c^{2}}\right)}}
$$

originates from the Lorentz factor $\gamma$ and has a calculated value of 1.

The relativistic mass $m$ is defined by $m=\gamma m_{0}$

The measured mean value was: $\quad Y=1.00037 \pm 0.00036$

The experimental set-up consisted in deflecting relativistic electrons on a path where the electrons were first deflected by magnetic forces and then by electrostatic forces. The electrostatic forces were adjusted until the electrons could be detected by the sensor and the electrostatic deflector was then calibrated using protons.

Many other experimental approaches have been carried out. Their measured results must include relativistic view effects as described by electromagnetism if the related forces have been used in the set-up. The important result is that the empirical evidence confirms the Lorentz transformation but no more than that.

Special Relativity defines energy using the following formula:

$$
E_{t}=\frac{m_{0} c^{2}}{\sqrt{\left(1-\frac{v^{2}}{c^{2}}\right)}}
$$

Equations [8] and [9] are equivalent and each can be calculated from the other equation.

Equation [9] is more compact and therefore more difficult to understand. It is usually interpreted as meaning that the speed of an object is limited to the speed of light except for trivial cases where no energy is transferred and that the total energy increases towards infinity the closer the path speed gets to the speed of light. This traditional interpretation must be closely examined given the arguments detailed in this paper.

As described for the Ehrenfest paradox, a rotation of the disc produces a relativistic view effect with contraction values depending on the path speed $v$. The same view effects can be obtained with a fixed disc and a rotating observer. A rotating observer will observe stars moving with a path speed of many times the speed of light. How could the rotation of an observer be the source of an infinite amount of energy? A rotation of an observer cannot be that, and the interpretation of Special Relativity must be wrong.

Relativistic view effects are specific to the transmission of information on the path of an object. The speed of information transfer is the speed of light applying to the line of sight of the observer. The calculation of such view effects is based on a Lorentz boost. The only contribution of a Lorentz boost is specific to the limitation of the speed of light in inertial frames. In particular it does not include any constraint applicable to the path speed $v$ or to the seen speed $v_{s}$. The supposition that they 
should be limited to the speed of light results from a strange interpretation of equation [9]. It is advisable to examine instead the equivalent equation [8] that gives more detailed information to understand relativity.

The total relativistic energy $E_{t}$ can be expanded in a Maclaurin series:

$$
E_{t}=m_{0} c^{2}+\frac{1}{2} m_{0} v^{2}+m_{0} c^{2}\left\{\frac{3}{8}\left(\frac{v}{c}\right)^{4}+\frac{5}{16}\left(\frac{v}{c}\right)^{6}+\cdots\right\}
$$

The term $m_{0} c^{2}$ is the rest mass energy and $\frac{1}{2} m_{0} v^{2}$ is the kinetic energy valid for both electromagnetism and classical mechanics including gravitation. The other terms in powers of $\frac{v}{c}$ starting from $\left(\frac{v}{c}\right)^{4}$ depend on the speed of light. They represent the relativistic view effects pertaining to electromagnetism and cannot contribute to energy.

The real energy $E_{\text {real }}$ is given by

$$
E_{\text {real }}=m_{0} c^{2}+\frac{1}{2} m_{0} v^{2}
$$

where $m_{0} c^{2}$ is the rest mass energy and $\frac{1}{2} m_{0} v^{2}$ is the kinetic energy specific to the observer.

The relativistic view effects $V E$ are represented by:

$$
V E=m_{0} c^{2}\left\{\frac{3}{8}\left(\frac{v}{c}\right)^{4}+\frac{5}{16}\left(\frac{v}{c}\right)^{6}+\cdots\right\}
$$

This equation describes non-existing energy supposed to originate from electromagnetism and matching path changes due to view effects.

The Maclaurin series is valid for equation [9] as well. That equation

$$
E_{t}=\frac{m_{0} c^{2}}{\sqrt{1-\frac{v^{2}}{c^{2}}}}
$$

gives a complex number with an imaginary part if the fraction $\frac{v}{c}$ is greater than one thereby limiting the speed $v$ to no more than the speed of light. This limitation needs not to apply to terms of the Maclaurin series not including the fraction $\frac{v}{c}$ if they describe a different entity. Energy and relativistic view effects apply to different entities. A path speed $v$ is used to calculate relativistic view effects and in that role it is limited to the speed of light. A seen speed $v_{s}$ is not used to calculate view effects and is free to be unlimited. A speed $v$ is not limited to the speed of light when applying to energy. The speed relevant for energy is the path speed $v$ as the seen speed $v_{s}$ is calculated for a Lorentz boost and includes view effects.

Equations [6] and [7] include relativistic view effects and result therefore in a confusing relationship of the speeds $v$ and $v_{s}$ due to different applicable speed ranges representing different entities. 
An object can have a path speed $v$ higher than the speed of light only if no other limitation applies. A photon is limited to the prevailing speed of light and this may apply to other particles such as neutrinos. Information on an object travelling faster than light will continue to be transferred by light and no faster than at the speed of light.

Depending on the case a photon will act as a carrier of information or as both an object and a carrier of information as for the deflection of light by the sun.

The formulas of energy [8] and [9] used by Special Relativity are based on the implicit assumption that electromagnetism would exclusively describe interactions using forces and exchanges of energy. This is not the case. Relativistic view effects are included as well, and they act with no force and no energy exchanges.

\section{On empirical evidence}

Empirical evidence has traditionally been considered as the supreme test of any theory in physics but will not represent the fundamental laws of nature whenever view effects induce a deformation of reality. Relativistic view effects are new to physics and their impact must be analyzed.

Relativistic view effects impact the images recorded by eyes or sensors. View effects exercise no force and do not exchange energy.

A first conclusion is that relativistic view effects must be excluded from the calculation of dark energy. Relativity does not contribute to energy.

The cosmic distance ladder may be impacted whenever measured or calculated distances include relativistic view effects. This may help explain dark matter and the discrepancies in the measurements of the Hubble constant. The impact of superluminal path speeds on events subject to extreme forces and amounts of energy, such as due to black holes, remains to be determined. A speed not limited to the speed of light may help validate the inflation period.

\section{On inertial behavior}

Inertia is tied to inertial frames and to inertial behavior. Mach considered that reference frames were inertial respective to the distribution of masses in the universe. The rest frame concept provides a simpler explanation.

In classical mechanics interactions of any sort use exchanges of energy including non-relativistic kinetic energy. Exchanges of energy explain inertial behavior. A body can be accelerated when absorbing energy from whatever source and can be decelerated when transferring energy to other bodies. An inertial reaction is proportional to the exchanged kinetic energy. There is no need to introduce yet unknown interactions. They would act in the same way. Relativistic view effects introduce a distortion of reality and do not contribute to inertial behavior.

Energy originating from any interaction can contribute to kinetic energy which depends on positive rest masses. This implies that an energy delivered by an interaction can be expressed as depending on such rest masses of bodies. It is therefore possible to use this dependence to describe the interactions of classical mechanics. Additional masses specific to interactions are redundant.

A photon with a rest mass of zero can be accelerated by gravitation as described in the section "On the deflection of light by the sun". This acceleration does not depend on the rest mass of the photon whose energy content must be determined with quantum mechanics as classical mechanics reaches its limits of validity at such small scales. 
The work $w$ is the amount of energy required to execute a task with:

$w=\int_{C} f \cdot d s$

$f$ is the force acting along a path C.

Then, using Newton's second law, we have:

$w=\int_{C} m_{i} a \cdot d s$

$a$ is the acceleration and $m_{i}$ is the inertial mass from Newton's second law.

Energy and work can be expressed as depending on the positive rest mass of bodies. This dependence applies as well to the right hand side of equation [10] and the inertial mass $m_{i}$ must be the rest mass $m_{0}$.

Calculations are always done in the rest frame of the observer and an energy impacting the observer will always be assigned to an impact on the body.

The rest frame of the observer ascertains the continuous existence of the inertial frame in which the laws of classical mechanics are valid including Newton's laws of gravitation. The laws of classical mechanics impacting the object in the rest frame of the observer are given by differential equations describing accelerated paths.

The rest frame of the object makes certain that it is always possible to cancel the impact of all interactions at any location of the object independently on whether inertial masses exist or not.

\section{References}

1) Blanchet Luc, Introduction à la Relativité Générale, Institut d'Astrophysique de Paris, UMR 7095 du CNRS; Université Pierre et Marie Curie, 75014 Paris, France

2) Hafele J. C., Keating Richard E., Around-the-World Atomic Clocks, Predicted Relativistic Time Gains and Observed Relativistic Time Gains, Science, New Series, Vol. 177, No,4044 (Jul. 14, 1972), pp. 166-170

3) Terrell James, Invisibility of the Lorentz Contraction, Los Alamos Scientific Laboratory, University of California, Phys, Rev. 116, 1041 - Published 15 November 1959

4) Mathpages, Bending Light, pp. 1-5, https://www.mathpages.com/rr/s6-03/6-03.htm

5) Will Clifford M., The 1919 measurement of the deflection of light, Department of Physics, University of Florida, Gainsville FL 32611, USA

6) Soares Domingos S.L., Newtonian gravitational deflection of light revised, Physics Department, ICEX, UFMG, C.P. 702, 30.123-970, Belo Horizonte, Brazil.

7) Einstein Albert, Relativity, The Special and General Theory, Digital Reprint, Elegant Books, p. 89

8) Meyer V., Reichart W., Staub, H.H., Experimentelle Untersuchung der Massen-Impulsrelation des Elektrons, Helvetia Physica Acta, Band 36 (1963), Heft VII, Persistenter Link: http://doi.org/10.5169/seals-113412

9) Reference (1), p.49 